\title{
EMI Developed Test Methodologies for Short Duration Noises
}

\author{
B. Karthik ${ }^{1 *}$ and T. V. U. Kiran Kumar ${ }^{2}$ \\ 'Assistant Professor, ECE Department, Bharath University, No:173, Agaram Road, Selaiyur, Chennai-73; karthiklect@gmail.com \\ 2Professor, ECE Department, Bharath University, No:173, Agaram Road, Selaiyur, Chennai-73; uday41@yahoo.com
}

\begin{abstract}
In addition to the functional aspects, Electromagnetic Compatibility (EMC) compliance of electronic devices is one of the important challenges that the developers encounter before the product release. Limiting the EM noise, both conducted and radiated is one of the main requirements to achieve this compliance. The methods defined in the traditional standards have got many shortcomings. Measurement of noise of short duration is an example. Some noises like the transients from a switching device also defeat the traditional measurement procedures. Conventional methods don't prove to be efficient for the measurement of such noises since it is difficult to capture the noise level for the whole frequency range within short duration using a single test receiver. In this paper, we have developed methods that can efficiently measure the short duration noise types mentioned above.
\end{abstract}

Keywords: EMC, EMI, Radiated, Emission, Short-Duration.

\section{Introduction}

Due to the rapid development of new electronic products and due to emerging new technologies, the ability to achieve compliance and to improve electromagnetic compatibility is a major challenge in development of electronic products. In a typical electromagnetic environment like railway where high current switching and electrical motors and high speed electronics are all required to work together and with communication systems, it is required to work without affecting other services external to it and also services internal to it. One of the challenging issues in such an environment is measuring the short duration noises that emerge as a result of arcing between the fixed power supply conductor and the train power collection device or due to any switching devices. Another issue associated with noise from a moving locomotive is that the worst case noise appears only when it is moving and will be available near the measuring antenna for a fraction of minute.

* Corresponding author:

B. Karthik (karthiklect@gmail.com)
Electromagnetic Compatibility (EMC) and Electromagnetic Interference (EMI) measures equipment which allow extracting comprehensive and accurate information within short measurement times will allow to reduce the costs and to improve the quality in circuit and system development. In conventional systems, radio noise and EMI are measured and characterized by super heterodyne radio receivers. The measurement is commonly termed as precompliance measurement and it includes both radiated and conducted emissions measurement. Pre-Compliance measurements of radiated emissions alone can take hours, since they must be performed between $30 \mathrm{MHz}$ and at least $1 \mathrm{GHz}$. In traditional measurement systems, the receivers are swept across the desired frequency range with a specified dwell time. To reliably detect a pulse-like disturbance, the observation time per frequency point must be at least as large as the reciprocal of its pulse rate. Therefore, we are proposing new techniques in this paper to estimate the short duration noises efficiently within short period of 
time. The techniques utilize a system with multiple detectors and also a time domain EMI measurement (TDEMI) system [1].

\section{The Proposed Method}

\subsection{System with Multiple Receivers}

In conventional measurement system, to cover the full frequency range $(9 \mathrm{kHz}$ to $1 \mathrm{GHz})$; measurements are to be made over several sub ranges using a single receiver or analyzer. For the case of commercial radiated emission measurement covering the range of $30 \mathrm{MHz}$ to $1 \mathrm{GHz}$ with an IF bandwidth of $120 \mathrm{kHz}$ and a step width of $40 \mathrm{kHz}$, to measure the entire spectrum with sufficient measurement accuracy, produces 24,250 frequency points. If the dwell time is $50 \mathrm{~ms}$ per frequency point, total measurement time for a single scan is nearly 20 minutes. The measurement will have to be repeated for different EUT (Equipment Under Test) angular orientations and different antenna heights making total measurement time extend to hours. Therefore, the short duration noise cannot be captured using traditional method. In order to make this measurement it is necessary to utilize multiple receivers measuring simultaneously.

For using multiple receivers, the noise is captured simultaneously using three different antennas. The measurement frequency range has been divided into three ranges based on the ranges of the antennae. The receivers are made to scan in the corresponding frequency range simultaneously. The data can be then logged to obtain a continuous plot.

The antennas used have been specified in Table 1.

\subsection{EMI Measurement in Time Domain}

In conventional EMI measurement systems the noise is recorded only when the noise frequency coincides with the current receiver scan frequency [2]. In the case of noise with transient pulses there is a higher probability

Table 1. Antennas \& frequency ranges

\begin{tabular}{ccc}
\hline S.No & Frequency Range & Antenna \\
\hline 1. & $9 \mathrm{kHz}-30 \mathrm{MHz}$ & $\begin{array}{c}\text { Active Monopole Antenna } \\
\text { (SAS-550-1B) }\end{array}$ \\
2. & $30 \mathrm{MHz}-230 \mathrm{MHz}$ & $\begin{array}{c}\text { Biconical Antenna } \\
\text { (R\&S HK116) }\end{array}$ \\
3. & $230 \mathrm{MHz}-1 \mathrm{GHz}$ & $\begin{array}{c}\text { Log-Periodic Antenna } \\
\text { (LPA-25) }\end{array}$ \\
\hline
\end{tabular}

that the noise is not captured. So we propose a measurement technique in which such noises will be captured using a digital oscilloscope with high memory and sampling rate. The oscilloscope is set to capture the data against a set trigger level. The samples thus obtained from the oscilloscope are converted to frequency domain via FFT to obtain the spectra. After FFT, the errors due to the frequency characteristics of antenna, transmission line, amplifier and anti-aliasing filter are corrected by signal processing. After this the analysis of Peak-, RMS-, Average- and Quasi-Peak values of the EMI signal is performed using appropriate digital filters [3]. A control computer has been used to gather data from the oscilloscope and perform the signal processing.

\section{Implementation of Measurement System with Multiple Receivers}

The considerations discussed in previous sections were taken into account in the process of developing the system with multiple receivers suitable for making improved emissions measurements. This system allows multiple receivers to be used to gather data over a period of time in each frequency range of interest [4].

\subsection{Measurement Setup}

Radiated emission measurements are generally measured from a EUT placed in an anechoic chamber where no noises other than that from the EUT will be present. The EUT for the proposed system will be a Noise Emitter. The noise from the EUT will be captured by the analyzers simultaneously such that the measurement should not cease while the download is underway. The analyzers are connected to a computer for the purpose of automation and data downloading. The block diagram of the total setup has been illustrated in Figure 1.

\subsection{Automation of Measurement}

The automation of the entire measurement system is necessary in order to avoid errors due to human interruptions. The In the proposed method we have used MATLAB for the purpose of instrument control and data downloading. The advanced features like Instrument Control toolbox and Graphical User Interface have been made use for the development of the software in MATLAB. 


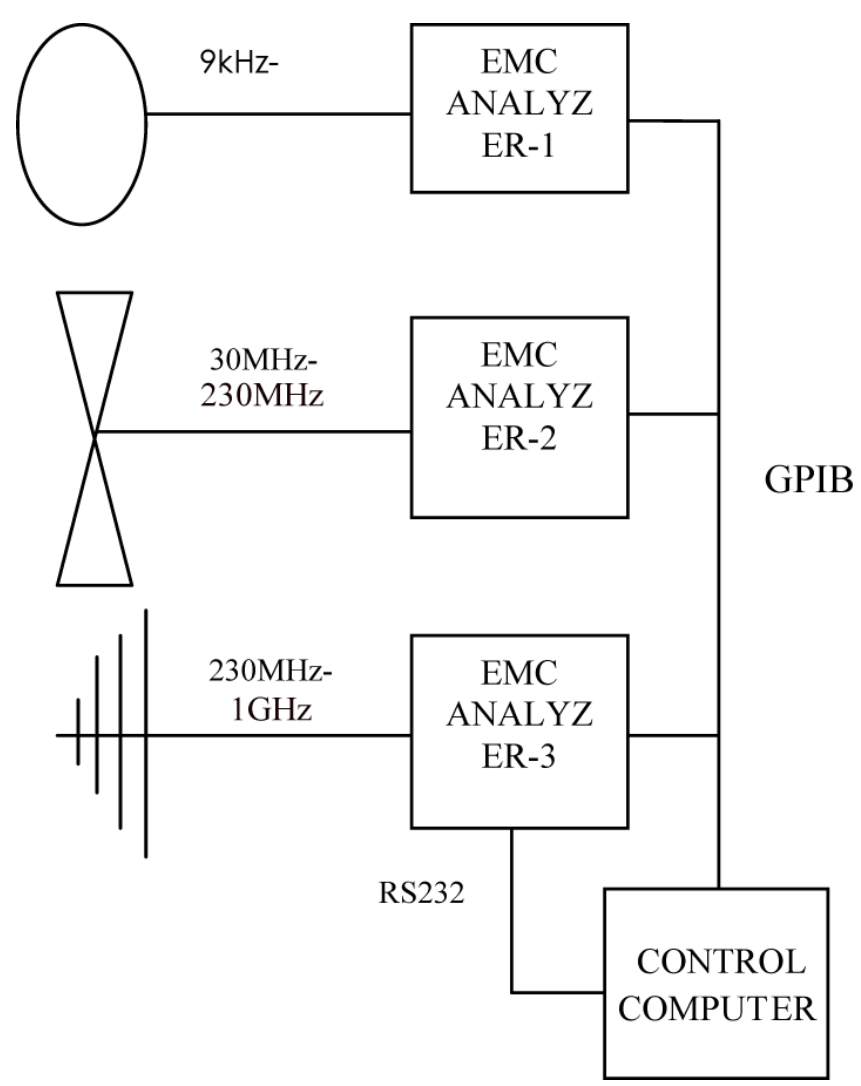

Figure 1. System with multiple analyzers.

The Instrument control toolbox provides a framework for communicating with the analyzer that supports

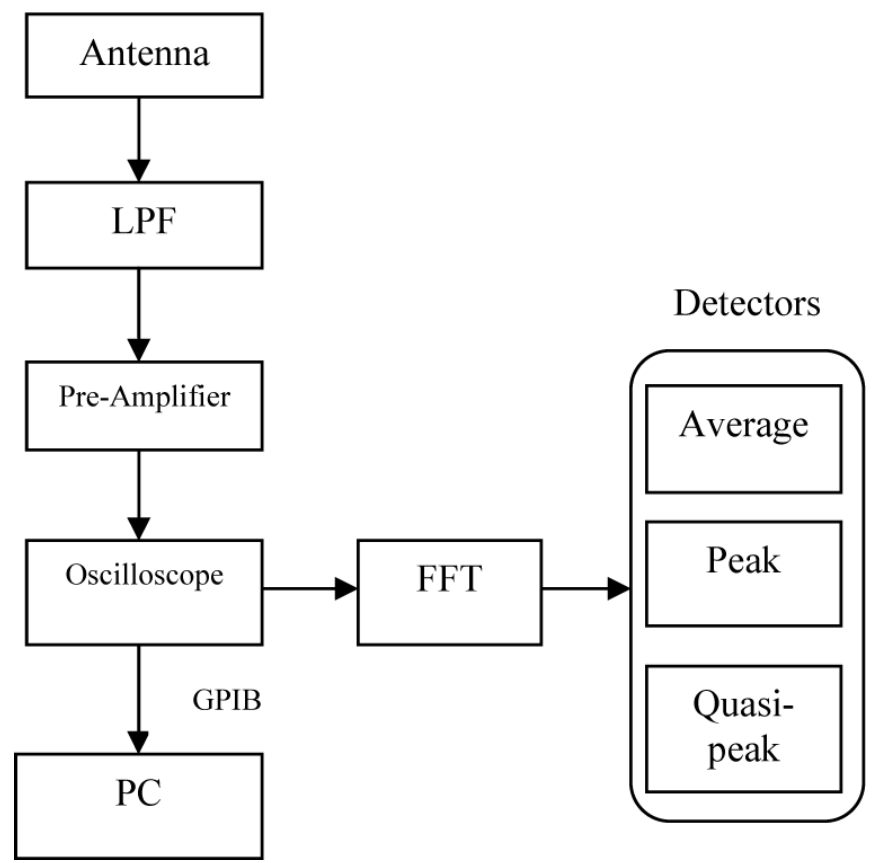

Figure 2. Time domain measurement system.

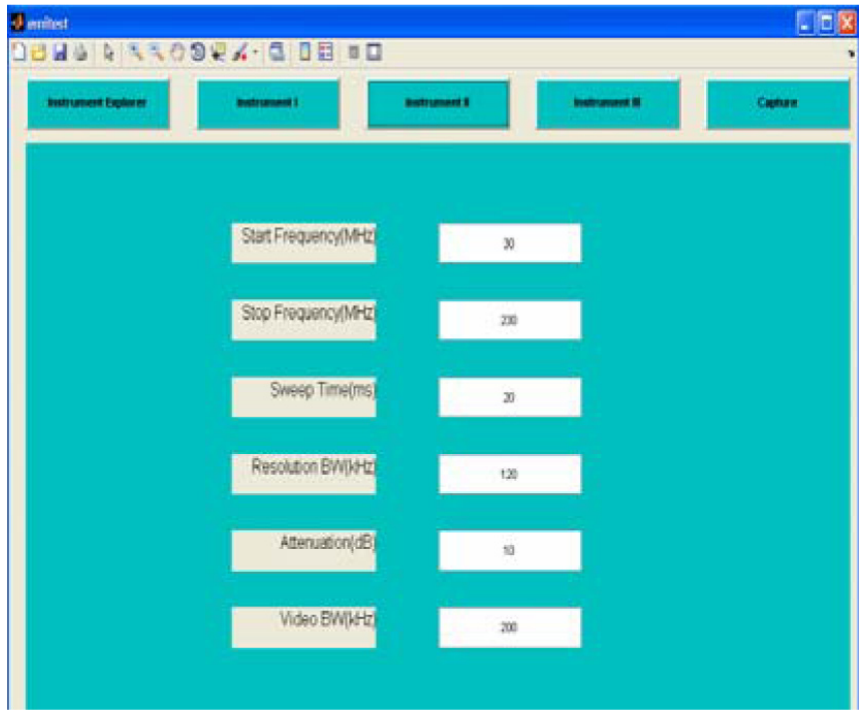

Figure 3. Measurement settings panel.

the GPIB interface protocols. GPIB driver software provided by Agilent has been installed to the system before making the interface and the GPIB commands were provided by Agilent to control the analyzer. GUI has been used in MATLAB to make the user interface easy. Figure 3 illustrates the GUI panel used for the measurement.

The front end has been designed in order to scan the instruments connected and to ensure the settings of the analyzer from the user end. The Resolution Bandwidth and Sweep time has been chosen as per the standard. The standard has been given in the Table 2 .

The settings for the three frequency ranges had been made in the corresponding panels. The analyzers are swept simultaneously and the trace is downloaded and finally a continuous plot is obtained using MATLAB. We have obtained a three-dimensional plot with many plots of frequency Vs. amplitude obtained at different times.

The results thus obtained are to be added with appropriate correction factors like cable loss and antenna factor for further validation. The amplitude value will be in $\mathrm{dB} \mu \mathrm{V} / \mathrm{m}$.

Table 2. Minimum sweep times and RBW as per CISPR

\begin{tabular}{cccc}
\hline S.No. & Frequency Range & $\mathbf{6 ~ d B}$ Bandwidth & Sweep Time \\
\hline 1. & $9 \mathrm{kHz}-150 \mathrm{kHz}$ & $200 \mathrm{~Hz}$ & $100 \mathrm{~ms} / \mathrm{kHz}$ \\
2. & $150 \mathrm{kHz}-30 \mathrm{MHz}$ & $9 \mathrm{kHz}$ & $100 \mathrm{~ms} / \mathrm{kHz}$ \\
3. & $30 \mathrm{MHz}-1 \mathrm{GHz}$ & $120 \mathrm{kHz}$ & $1 \mathrm{~ms} / \mathrm{MHz}$ \\
\hline
\end{tabular}




\section{Transient Signal Detection}

Traditional EMC tests make higher probability for transient signals to be ignored. They provide relatively minor annoyance to the earlier EMI issues such as disruption to analog based radio transmissions [1]. This is becoming a less valid assumption as increasing numbers of broadband digital modulation techniques are coming into use in radio communication systems. So the developed measurement system should also be able to quantify the transient activity. It is possible with the time domain measurement system we have proposed.

\subsection{Measurement Setup}

The measurements will be made in the laboratory to characterise the emissions from a representative arc. The EUT to be tested is placed in an anechoic room with appropriate wideband antenna at specified distance from the equipment and connected to the input of a sampling oscilloscope. The entire setup has been illustrated in Figure 2.

The obtained signal will be converted into frequency domain by performing DFT and further processed to get a smooth signal. After that Quasi-peak detection has to be made for further validation.

\subsection{Frequency Domain Estimation}

The signal from an oscilloscope is a time domain signal and it should be converted into frequency domain using DFT (Discrete Fourier Transform). DFT computes the amplitude spectrum a time record of length $\mathrm{N}$. So the time record $x[n]$ needs to be multiplied with a window $w[n]$ of length $\mathrm{N}$ to avoid spectral leakage:

$$
y[n]=x[n] w[n]
$$

The window function is equivalent to the bandwidth of the Intermediate-Frequency-filter (IF-filter) of the conventional EMI-Receiver. A CISPR bandpass filter has been designed to meet the requirement with the following impulse function [2]:

$$
h(t)=4 \omega_{a} e^{-\omega_{a} t}\left(\sin \omega_{a} t-\omega_{a} t \cos \omega_{a} t\right) \cos \omega_{0} t
$$

The spectral estimation of time domain signal should not take a long time in the process. Therefore we go for a Fast Fourier Transform (FFT) [5]. The sampling frequency of the time-domain signal must be chosen properly to minimize the error between the digital and the analog implementation. The FFT contains information between
0 and fs, however, we know that the sampling frequency must be at least twice the highest frequency component. The DFT being applied to each of the data blocks and is defined as follows:

$$
Z[k]=\sum_{n=0}^{N-1} z[n] e^{-j \frac{2 \pi k n}{N}} \quad n=1, \ldots, N ; k=1, \ldots, N
$$

The signal should then be processed into a quasi-peak detector. The quasi-peak detector has a charge rate much faster than the discharge rate; therefore the higher the repetition rates of the signal the higher the output of the quasi-peak detector. The quasi-peak detector also responds to different amplitude signals in a linear fashion. High amplitude, low repetition rate signals could produce the same output as low amplitude high repetition rate signals.

\section{Measurement Results}

The measurement results obtained from the EMC analyzer and plotted using MATLAB is shown in the Figure 4. The advantage of using MATLAB for plotting is that the plot can be zoomed or rotated to get a clear picture. The impulse response of the CISPR bandpass filter designed has been shown in Figure 5. Figure 6 shows the digital Quasi-peak detector output.

\section{Conclusion}

The proposed method has proved efficient for the measurement of the short duration noises and transients since it

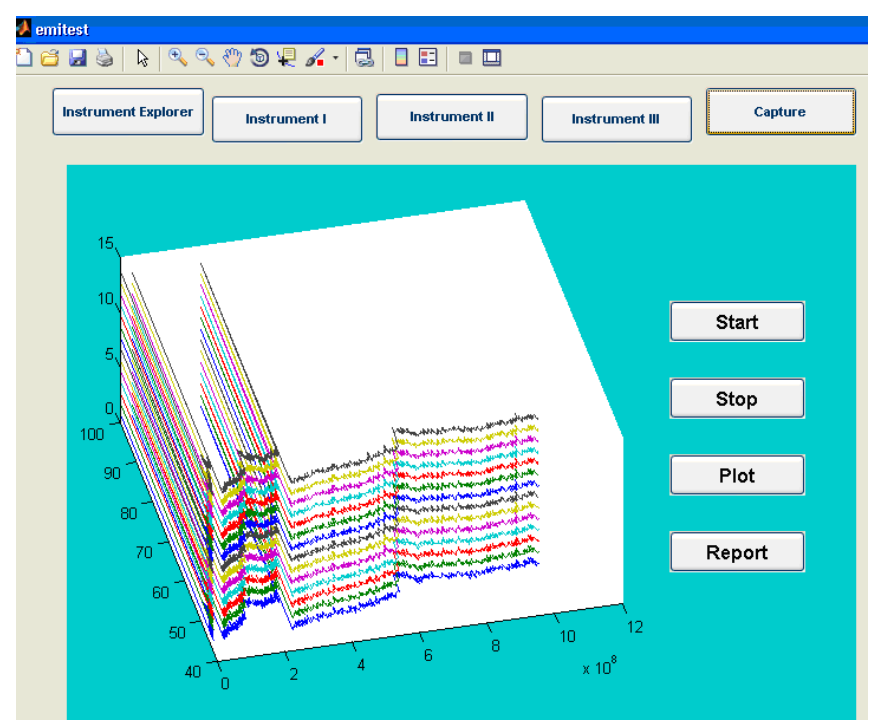

Figure 4. Plot obtained from spectrum analyzer. 

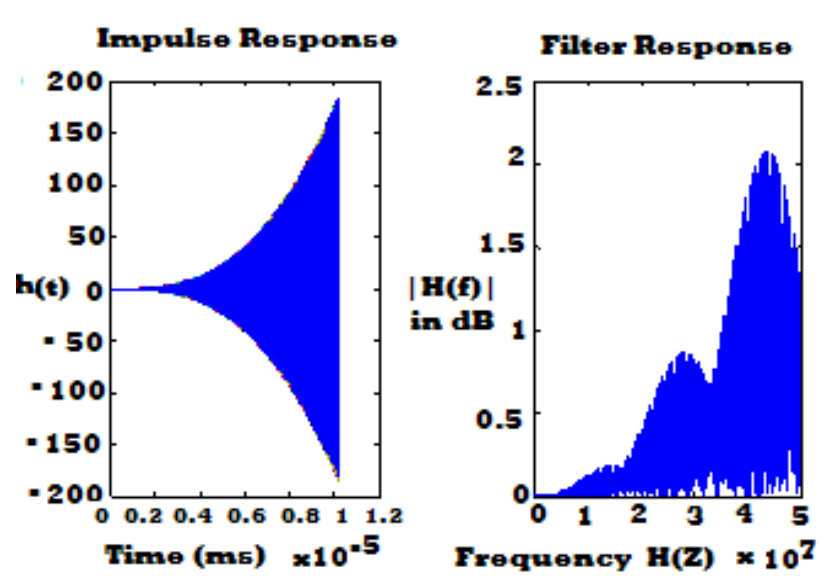

Figure 5. CISPR bandpass filter \& Impulseresponse.

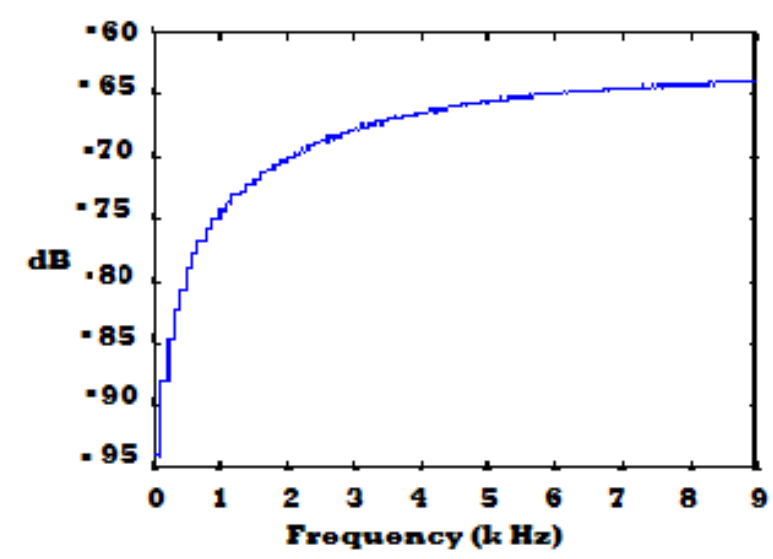

Figure 6. Signal vs. quasi-peak detector output. takes only short duration for the measurement of the signal over a wide range of frequency. Using time domain measurement system the measurement can be reduced by a factor of 10 .

\section{References}

1. Rowell A J, Bozec D et al. (2004). Improved methods for the measurement of radio frequency emissions from railways, York EMC Services Ltd, University of York, Heslington.

2. Braun S, Donauer T et al. (2008). A real-time time-domain EMI measurement system for full-compliance measurements according to CISPR 16-1-1, IEEE Transactions on Electromagnetic Compatibility, vol 50(2), 259-267.

3. Krug F, and Russer P (2006). A novel time-domain electromagnetic interference (TDEMI) measurement system, Institute for High-Frequency Engineering, Technische Universität München, Arcisstrasse 21, 80333 Munich, Germany.

4. Bellan D, and Gaggelli A (2009). Noise effects in timedomain systems involving three-axial field probes for the measurement of non-stationary radiated emissions, IEEE Transactions On Electromagnetic Compatibility, vol 51, No. 2, 192-203.

5. Krug F, and Russer P (2002). Ultra-fast broadband EMI measurement in time domain using FFT and Periodogram, IEEE International Symposium On Electromagnetic Compatibility Digest, Minneapolis, USA, 577-582. 